\title{
Supporting Information for \\ Global analysis of fluorometric titration curves in the presence of excited-state association and quenching
}

\author{
Eugene Novikov ${ }^{a}$ and Noël Boens ${ }^{\star}, b$
}

${ }^{a}$ Institut Curie, Service Bioinformatique, 26 Rue d'Ulm, Paris Cedex 05, 75248

France

${ }^{b}$ Department of Chemistry, Katholieke Universiteit Leuven, Celestijnenlaan 200fbus 02404, 3001 Heverlee, Belgium

The Supporting Information contains the Maple scripts used in the main paper.

1. Ground-state dissociation constant $K_{d}$ from a single fluorometric titration curve (eq 14)

$>$ restart;

$>$ with(linalg):

Set of equations for the rate constants (eq 11)

$>\mathrm{x} 0$ : $=\mathbf{k d} * \mathrm{k} 01 *(\mathrm{k} 02+\mathrm{k} 12)$

$>\mathrm{x} 1:=(\mathrm{kd} *(\mathrm{k} 01 * \mathrm{kq} 2+\mathrm{k} 21 * \mathrm{k} 02)+(\mathrm{k} 01+\mathrm{kd} * \mathrm{kq} 1) *(\mathrm{k} 02+\mathrm{k} 12)) / \mathrm{x} 0$

$>\mathrm{x} 2:=(\mathrm{k01} * \mathrm{kq} 2+\mathrm{kq} 1 * \mathrm{k} 12+(\mathrm{kd} * \mathrm{kq} 2+\mathrm{k02}) *(\mathrm{kq} 1+\mathrm{k} 21)) / \mathrm{x} 0$;

$>\mathrm{x} 3:=\mathrm{kq} 2 *(\mathrm{kq} 1+\mathrm{k} 21) / \mathrm{x} 0$;

Roots of the third-degree polynomial (eq 13)

$>$ solve $\left(\left\{x 3 * Z^{\wedge} 3-x 2 * Z^{\wedge} 2+x 1 * Z Z-1=0\right\},\left\{\_Z\right\}\right)$;

2. Identifiability of the ground-state dissociation constant $K_{d}$

$>$ restart;

$>$ with(linalg):

Set of equations for the rate constants (eq 11)

$>\mathrm{x} 0$ : $=\mathrm{kd} * \mathrm{k} 01 *(\mathrm{k} 02+\mathrm{k} 12)$

$>$ eq1 := $(\mathrm{kd} *(\mathrm{k} 01 * \mathrm{kq} 2+\mathrm{k} 21 * \mathrm{k} 02)+(\mathrm{k} 01+\mathrm{kd} * \mathrm{kq} 1) *(\mathrm{k} 02+\mathrm{k} 12)) / \mathrm{x} 0=\mathrm{x} 1$;

$>$ eq2 $:=(\mathrm{k} 01 * \mathrm{kq} 2+\mathrm{kq} 1 * \mathrm{k} 12+(\mathrm{kd} * \mathrm{kq} 2+\mathrm{k02}) *(\mathrm{kq} 1+\mathrm{k} 21)) / \mathrm{x} 0=\mathrm{x} 2$;

$>$ eq $3:=\mathrm{kq} 2 *(\mathrm{kq} 1+\mathrm{k} 21) / \mathrm{x} 0=\mathrm{x} 3$;

Set of equations for the alternative rate constants (eq 16)

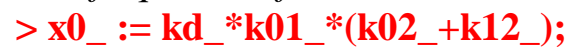

$>\mathrm{x} 1:=\left(\mathrm{kd} \mathrm{C}_{-} *\left(\mathrm{k} 01 \_* \mathrm{kq} 2 \_+\mathrm{k} 21 \_* \mathrm{k} 02 \_\right)+\left(\mathrm{k} 01 \_+\mathrm{kd} \_* \mathrm{kq} 1 \_\right) *\left(\mathrm{k} 02 \_+\mathrm{k} 12 \_\right)\right) / \mathrm{x} 0 \_$;

$>\mathrm{x} 2:=(\mathrm{k} 01$ _*kq2_+kq1_*k12_+(kd_*kq2_+k02_)*(kq1_+k21_ $)) / \mathrm{x} 0 \_$;

$>\mathrm{x} 3$ := kq2_*(kq1_+k21_)/x0_;

Combine eqs 15 a and $15 b$ at one emission wavelength and (at least two) different excitation wavelengths

$>\mathrm{eq} 41:=(\mathrm{kq} 2+\mathrm{t} 1 * \mathrm{k} 21) /(\mathrm{k} 02+\mathrm{k} 12)=\left(\mathrm{kq} 2 \_+\mathrm{t} 1 \_* \mathrm{k} 21 \_\right) /\left(\mathrm{k} 02 \_+\mathrm{k} 12 \_\right)$;

Combine eqs $15 b$ and $15 c$ at the same emission wavelength and (at least two)

different excitation wavelengths

\footnotetext{
* To whom correspondence should be addressed. E-mail: Noel.Boens@ chem.kuleuven.be, Fax: +3216-327990.
} 
$>$ eq51 := $(\mathrm{k} 01+\mathrm{k} 12 / \mathrm{t} 1) /(\mathrm{kq} 1+\mathrm{k} 21)=\left(\mathrm{k} 01 \_+\mathrm{k} 12 \_/ \mathrm{t} 1 \_\right) /\left(\mathrm{kq} 1 \_+\mathrm{k} 21 \_\right)$;

Combine eqs $15 a$ and $15 b$ at another emission wavelength and (at least two) different excitation wavelengths

$>$ eq42 := $(\mathrm{kq} 2+\mathrm{t} 2 * \mathrm{k} 21) /(\mathrm{k} 02+\mathrm{k} 12)=\left(\mathrm{kq} 2 \_+\mathrm{t} 2 \_* \mathrm{k} 21 \_\right) /\left(\mathrm{k} 02 \_+\mathrm{k} 12 \_\right)$

Combine eqs $15 \mathrm{~b}$ and $15 \mathrm{c}$ at the same emission wavelength and (at least two)

different excitation wavelengths

$>$ eq52 := $(\mathrm{k} 01+\mathrm{k} 12 / \mathrm{t} 2) /(\mathrm{kq} 1+\mathrm{k} 21)=\left(\mathrm{k} 01++\mathrm{k} 12 \_/ \mathrm{t} 2 \_\right) /\left(\mathrm{kq} 1 \_+\mathrm{k} 21 \_\right)$;

Solution of the obtained set of algebraic equations

$>\mathrm{w}:=\operatorname{solve}(\{$ eq1,eq2,eq3,eq41,eq51,eq42,eq52\},

$\{\mathrm{k} 01, \mathrm{k02}, \mathrm{k12}, \mathrm{k21}, \mathrm{kd}, \mathrm{kq} 1, \mathrm{kq} 2, \mathrm{t1}, \mathrm{t} 2\})$;

3. Determination of the ground-state dissociation constant $K_{d}$, (eqs 17 and 25)

$>$ restart:

$>$ with(linalg):

Elimination of the coefficients e (epsilon) and $c$ in eq 10

Define a new variable $x:=e 1 / e 2$, the excitation ratio taken at a certain excitation wavelength

Equation 10a with the explicit dependence on the emission wavelength

$>$ eq0 $:=\mathbf{i}->\mathbf{y} 0 . \mathbf{i}=\mathbf{x}^{*} \mathbf{e} 2 * \mathbf{c} 1 . \mathbf{i} / \mathbf{k 0 1}$

Equation $10 b$ with the explicit dependence on the emission wavelength

$>$ eq1 : $=$ i-

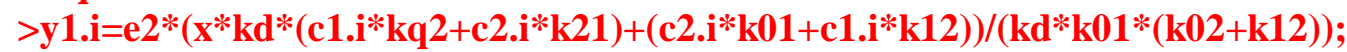

Equation 10c with the explicit dependence on the emission wavelength

$>$ eq2 := i->y2.i=e $2 * \mathrm{c} 2 . \mathrm{i} *(\mathrm{kq} 1+\mathrm{k} 21) /(\mathrm{kd} * \mathrm{k} 01 *(\mathrm{k} 02+\mathrm{k} 12))$;

Extract the coefficients $\mathrm{cl}$ and $\mathrm{c} 2$ from eqs $10 \mathrm{a}$ and $10 \mathrm{c}$ at two different emission

wavelengths

$>\operatorname{assign}(\operatorname{solve}(\operatorname{eq0}(1),\{\mathrm{c} 11\})) ; \operatorname{assign}(\operatorname{solve}(\operatorname{eq} 2(1),\{\mathrm{c} 21\}))$;

$\operatorname{assign}(\operatorname{solve}(\operatorname{eq} 0(2),\{\mathrm{c} 12\}))$; $\operatorname{assign}(\operatorname{solve}(\mathbf{e q} 2(2),\{\mathrm{c} 22\}))$;

Substitute the obtained coefficients in eq $10 \mathrm{~b}$

$>$ eu1 := collect(factor(eq1(1)),[y01,y21,x]); eu2 :=

collect(factor(eq1(2)),[y02,y22,x]);

To simplify derivations, define a set of new variables, incorporating the rate constants

and the excitation ratio $x$

$>\mathrm{s}:=\{(\mathrm{kd} * \mathrm{kq} 2 * \mathrm{kq} 1+\mathrm{kd} * \mathrm{kq} 2 * \mathrm{k} 21) /((\mathrm{kq} 1+\mathrm{k} 21) * \mathrm{kd} *(\mathrm{k} 02+\mathrm{k} 12))+$

$(\mathrm{k} 12 * \mathrm{kq} 1+\mathrm{k} 12 * \mathrm{k} 21) /((\mathrm{kq} 1+\mathrm{k} 21) * \mathrm{kd} *(\mathrm{k} 02+\mathrm{k} 12)) / \mathrm{x}=\mathrm{z0}$,

$\left(\mathbf{k d}^{\wedge} 2 * \mathbf{k} 21 * \mathrm{k} 02+\mathrm{kd}^{\wedge} 2 * \mathbf{k} 21 * \mathrm{k} 12\right) * \mathrm{x} /((\mathrm{kq} 1+\mathrm{k} 21) * \mathbf{k d} *(\mathbf{k} 02+\mathrm{k} 12))+$

$(\mathbf{k d} * \mathbf{k} 01 * \mathbf{k} 02+\mathbf{k d} * \mathbf{k} 01 * \mathbf{k} 12) /((\mathbf{k q 1}+\mathbf{k} 21) * \mathbf{k d} *(\mathbf{k} 02+\mathrm{k} 12))=\mathrm{z} 1\}$

Solve the simplified set of equations with respect to the new variables z0 and z1

$>\operatorname{solve}(\{\operatorname{subs}(\mathrm{s}, \mathrm{eu} 1), \operatorname{subs}(\mathrm{s}, \mathrm{eu} 2)\},\{\mathrm{z} 0, \mathrm{z} 1\})$;

Eliminate $x$ from $z 0$ and $z 1(*)$

$>$ collect(factor(solve $(\mathbf{s},\{\mathbf{x}, \mathrm{z} 0\})),[\mathrm{z} 1, \mathbf{k q} 2])$;

The same relations can be obtained for another excitation wavelength

$>$ restart;

$>$ with(linalg):

Determination of the ground-state dissociation constant Kd (eqs 17 and 25)

Set of equations for the rate constants (eq 11)

$>\mathrm{x} 0$ : = kd*k01*(k02+k12);

$>\mathrm{eq} 1:=(\mathrm{kd} *(\mathrm{k} 01 * \mathrm{kq} 2+\mathrm{k} 21 * \mathrm{k} 02)+(\mathrm{k} 01+\mathrm{kd} * \mathrm{kq} 1) *(\mathrm{k} 02+\mathrm{k} 12)) / \mathrm{x} 0=\mathrm{x} 1$;

$>$ eq2 := (k01*kq2+kq1*k12+(kd*kq2+k02)*(kq1+k21))/x0=x2; 
$>\mathrm{eq} 3:=\mathrm{kq} 2 *(\mathrm{kq} 1+\mathrm{k} 21) / \mathrm{x} 0=\mathrm{x} 3$

Additional equations from eqs 10 and 19 written for two excitation wavelengths

[see also (*) from Elimination of the coefficients e (epsilon) and $c$ in eq 10]

$>$ eq4 := z01*(k02+k12)=kq2+k12*k21/(z11*(kq1+k21)-k01); eq5 :=

$\mathrm{z02} *(\mathrm{k} 02+\mathrm{k} 12)=\mathrm{kq} 2+\mathrm{k} 12 * \mathrm{k} 21 /(\mathrm{z} 12 *(\mathrm{kq} 1+\mathrm{k} 21)-\mathrm{k} 01)$;

Solve a set of 5 equations eq1, ... , eq 5

$>\operatorname{assign}(\operatorname{solve}(\{$ eq1,eq4,eq5 $\},\{k 12, \mathrm{kq} 2, \mathrm{k} 21\}))$ :

$>$ eq2:=algsubs $\left(k^{\wedge}{ }^{\wedge} 2=c e\right.$, factor $\left.(\mathrm{eq} 2)^{*} k d^{\wedge} 2\right)$ :

$>$ eq3:=algsubs $\left(k^{\wedge}{ }^{\wedge} 2=c e\right.$, factor $\left.(e q 3)^{*} k d^{\wedge} 2\right)$ :

$>\operatorname{assign}(\operatorname{solve}(\{$ eq2,eq 3$\},\{\mathrm{kd}, \mathrm{ce}\}))$;

The final result for the ground-state dissociation constant $\mathrm{Kd}$ (eq 17)

$>\mathbf{k d}:=\operatorname{collect}(\mathbf{f a c t o r}(\mathbf{k d}),[\mathbf{x} 1, \mathbf{x} 2, \mathbf{x} 3])$;

Check the result using the coefficients p1, p2 and p3 (eq 18)

$>\mathrm{p} 1:=\mathrm{z} 01 * \mathrm{z} 11-\mathrm{z} 02 * \mathrm{z} 12$;

$>\mathrm{p} 2:=(\mathrm{z} 02-\mathrm{z01}) * \mathrm{z} 11 * \mathrm{z} 12+\mathrm{z} 12-\mathrm{z} 11$

$>\mathrm{p} 3:=(\mathrm{z} 11-\mathrm{z} 12) * \mathrm{z} 02 * \mathrm{z} 01-\mathrm{z} 02+\mathrm{z} 01$

Reproduce eq 17

$>\mathrm{kd}_{-}:=\left(\mathrm{p} 1^{\wedge} 2 * \mathrm{x} 1+\mathrm{p} 2 * \mathrm{p} 1 * \mathrm{x} 2+\mathrm{p} 2{ }^{\wedge} 2 * \times 3-\right.$

$\mathrm{p} 3 * \mathrm{p} 1) /\left(\mathrm{p} 1{ }^{\wedge} 2 * \mathrm{x} 1 \wedge 2+\left(\mathrm{p} 2 * \mathrm{p} 1 * \mathrm{x} 2+\mathrm{p} 2{ }^{\wedge} 2 * \mathrm{x} 3-2 * \mathrm{p} 3 * \mathrm{p} 1\right) * \mathrm{x} 1-\right.$

$\left.\mathrm{p} 2 * \mathrm{p} 3 * \mathrm{x} 2+\mathrm{p} 2 * \mathrm{p} 1 * \mathrm{x} 3+\mathrm{p} 3^{\wedge} 2\right)$;

Expect 0 - no difference

> factor(kd-kd_);

Model without quenching (eq 25)

$>$ factor $(\operatorname{eval}(k d, x 3=0))$; 